\title{
Glycated albumin in pregnancy: reference intervals establishment and its predictive value in adverse pregnancy outcomes
}

\author{
Ying Dong ${ }^{1}$, Yanhong Zhai ${ }^{1}$, Jing Wang ${ }^{1}$, Yi Chen², Xin Xie ${ }^{1}$, Chunhong Zhang ${ }^{1}$, Jingrui Liu ${ }^{1}$, Yifan Lu', \\ Guodong Tang ${ }^{1,3}$, Lican Han ${ }^{1}$, Lin $\mathrm{Li}^{4}$ and Zheng Cao ${ }^{1 *}$
}

\begin{abstract}
Background: Many efforts have been focused on the alternative glycemic marker glycated albumin (GlyA) and its application in pregnancy during which profound physiological changes take place. Our objective was to determine the reference intervals (RIs) of GlyA in healthy Chinese pregnant women and to assess the predictive value of serum GlyA in adverse pregnancy outcomes.

Methods: Totally 421 healthy subjects including 137 in the first trimester, 152 in the second trimester, and 132 in the third trimester were enrolled from March to July 2019, for the purpose of establishing the trimester-specific RIs of GlyA. In addition, 67 pregnant women diagnosed with GDM were enrolled at 24-28 weeks of gestation. The diagnostic value of GlyA for GDM patients was evaluated and compared with that of fasting plasma glucose (FPG) at 24-28 weeks of gestation. The association between GlyA in the late pregnancy and the adverse pregnancy outcomes was analyzed with the data collected from January to June 2018 at our hospital.

Results: The estimated RIs of GlyA in present study were $11.26-15.10 \%, 10.04-13.50 \%$, and $9.76-13.09 \%$ in the first, second, and third trimesters respectively. The areas under receiver operating characteristic (ROC) curves were 0.503 for GlyA and 0.705 for FPG. More importantly, the GlyA level in the third trimester was not more elevated in the patients with adverse pregnancy outcomes including large for gestational age (LGA), preterm delivery, hypertension and preeclampsia (PE). The exception was made with the GDM patients who suffered from postpartum hemorrhage and had significantly higher GlyA levels than the control group.

Conclusions: Our results showed that the GlyA was continuously decreased as the gestational age went up. The GlyA testing has limited value in diagnosing GDM and predicting adverse pregnancy outcomes.
\end{abstract}

Keywords: Glycated albumin, Gestational diabetes mellitus, Adverse pregnancy outcomes, Birth weight

\section{Background}

Gestational diabetes mellitus (GDM) is defined as diabetes diagnosed in the second or third trimester of pregnancy that was not clearly overt diabetes before pregnancy [1]. As one of the most common pregnant complications, the prevalence of GDM has been increased during the last decades and is estimated to continue to increase in the future [2]. According to a

\footnotetext{
* Correspondence: zhengcao2011@hotmail.com

${ }^{1}$ Department of Laboratory Medicine, Beijing Obstetrics and Gynecology Hospital, Capital Medical University, 251 Yaojiayuan Road, Beijing 100026, China

Full list of author information is available at the end of the article
}

research based on over 125 million pregnant subjects between 1979 and 2010, the increased prevalence of GDM can be mainly attributed to high maternal age and body mass index (BMI) [3]. Adverse pregnancy outcomes of GDM affect both mothers and newborns in short and long terms. The women who were diagnosed with GDM have higher risk of type 2 diabetes and cardiovascular diseases after delivery [4]. In addition, GDM is closely associated with metabolism disorders, hypertensive disorders, preeclampsia (PE), large for gestational age (LGA, birth weight above the 90th percentile for gestational age), cesarean delivery and related birth injury in perinatal period $[3,4]$.

(c) The Author(s). 2020 Open Access This article is distributed under the terms of the Creative Commons Attribution 4.0 International License (http://creativecommons.org/licenses/by/4.0/), which permits unrestricted use, distribution, and reproduction in any medium, provided you give appropriate credit to the original author(s) and the source, provide a link to the Creative Commons license, and indicate if changes were made. The Creative Commons Public Domain Dedication waiver (http://creativecommons.org/publicdomain/zero/1.0/) applies to the data made available in this article, unless otherwise stated. 
Strict glycemic control is the key to prevent or decrease adverse perinatal complications. With a half-life of 8-12 weeks for red blood cells, the glycated hemoglobin A1c (HbA1c) can reflect ambient blood glucose level in the past 2-3 months [5]. It has been widely used in monitoring glycemic state and guiding clinical therapy for diabetes patients. The HbA1c threshold of $6.5 \%$ for diabetes mellitus diagnosis is supported by the DETECT-2 collaboration [6]. However, the glycemic status may not be accurately monitored by the HbA1c level in some situations such as hemolytic anemia, iron deficiency anemia, uremia, hemoglobinopathies and pregnancy [7]. In normal pregnancy, the HbAlc level presents biphasic changes, including a significant decrease in the second trimester and a slowly elevation to its peak level in the third trimester [8]. For example, Richard et al. found that the HbAlc concentration reached the nadir level at the 24 week's gestation [9]. Alternatively, glycated albumin (GlyA) is formed through a nonenzymatic reaction between blood glucose and serum albumin. As not affected by hemoglobin metabolism or iron-deficient anemia, GlyA is often recommended for clinical practice on glycemic control in pregnancy where HbA1c is not appropriate to implement $[7,10]$.

Only a few articles focused on establishing the GlyA reference ranges in pregnancy. In a multi-center study based on the Japanese population, the GlyA level was significantly decreased in the second and the third trimesters compared with the first trimester [8]. In another study with 1479 normal pregnant women, the mean levels of GlyA were $11.53 \%$ in the $24-28$ weeks and $10.23 \%$ in the $36-38$ weeks [11]. According to the above studies in which the GlyA level was significantly decreased during pregnancy, it is important to establish trimester-specific reference intervals (RIs). Moreover, it is still controversial if higher GlyA level is associated with maternal and complications in GDM patients $[12,13]$. The purpose of this study was to determine the RIs of GlyA in Chinese pregnant women, and to assess the predictive value of GlyA in adverse pregnancy outcomes.

\section{Methods}

\section{Study population}

For the GlyA RIs establishment, a cross-section study was performed. Any patient that had already been recruited was not enrolled for the second time during the entire sample collection period. Totally 605 singleton pregnant women attending routine check-ups in the Beijing Obstetrics and Gynecology Hospital from March to July 2019 were initially recruited. The following exclusion criteria were applied in our study: pre-pregnancy BMI $\geq 25 \mathrm{~kg} / \mathrm{m}^{2}$ [14], liver dysfunction with elevated transaminases, abnormal kidney function with elevated $\mathrm{Cr}$ and Bun, serum albumin lower than $32 \mathrm{~g} / \mathrm{L}$, elevated fasting plasma glucose (FPG) (normal range of $3.9-6.1 \mathrm{mmol} /$ $\mathrm{L}$ in the first trimester) or GDM, lipid metabolism disorders, clinical or subclinical thyroid dysfunction. Eventually, 421 out of 605 healthy subjects were enrolled for RIs establishment, including 137 in the first trimester, 152 in the second trimester, and 132 in the third trimester. A sample size of more than 120 was used to derive the RI by the nonparametric approach according to the Clinical and Laboratory Standards Institute (CLSI) guideline EP28A3C [15]. The demographic characteristics of healthy subjects in three trimesters were shown in Table 1.

In present study, all the GDM patents were diagnosed by the 75-g oral glucose tolerance test (OGTT) according to the International Association of Diabetes and Pregnancy study Groups (IADPSG) 2010 criteria [16]). Specifically, GDM was determined by meeting one of the three following criteria: FPG $\geq 5.1 \mathrm{mmol} / \mathrm{L}, 1$-h postprandial blood glucose $\geq 10.0 \mathrm{mmol} / \mathrm{L}$, or 2 -h postprandial blood glucose $\geq 8.5 \mathrm{mmol} / \mathrm{L}$. There were 67 pregnant women diagnosed with GDM and enrolled at 24-28 weeks of gestation. The collected serum samples of the recruited healthy and GDM subjects were stored at $80^{\circ} \mathrm{C}$ before the GlyA testing.

In addition, 894 pregnant subjects that were diagnosed with GDM between January and June 2018 and delivered live newborns at our hospital were analyzed in the association study with the GlyA values of the third trimester and adverse pregnant outcomes, including preterm delivery, postpartum hemorrhage, $\mathrm{PE}$, hypertension and LGA. Meanwhile, 327 pregnant women without GDM or pre-pregnant DM were recruited in the third trimester as non-GDM group, with their sera samples tested for GlyA and pregnancy outcomes recorded after delivery. A schematic diagram was made for illustrating the flow of participants and study design (Fig. 1).

\section{GlyA measurement}

The GlyA results were calculated as the ratio of glycated albumin over albumin. Specifically, the glycated albumin and albumin were assayed on the fully automated biochemical analyzer Abbott C16000 (Abbott Park, IL, USA) using the peroxidase method (Glycated albumin assay kit, Catalog Number 3R43.09, Beijing Strong Biotechnologies Inc., Beijing, China). Total albumin was quantified by the bromocresol purple method (Glycated albumin assay kit, Catalog Number 3R43.10, Beijing Strong Biotechnologies Inc., Beijing, China).

\section{Statistical analysis}

The Dixon method was applied to remove the outliers from the dataset in the RI study. The numerical data of GlyA level was presented as the mean \pm standard 
Table 1 Characteristics of healthy pregnant women in three trimesters

\begin{tabular}{llll}
\hline & First trimester & Second trimester & Third trimester \\
\hline Number & 137 & 152 & 132 \\
Age (years) & $30.84 \pm 3.19$ & $31.04 \pm 3.37$ & $30.94 \pm 4.76$ \\
Pre-pregnancy BMI $\left(\mathrm{kg} / \mathrm{m}^{2}\right)$ & $21.01 \pm 2.19$ & $20.28 \pm 1.97$ & $20.87 \pm 1.95$ \\
Gestational age (weeks) & $6.81 \pm 1.11$ & $23.30 \pm 2.44$ & $31.79 \pm 0.83$ \\
\hline
\end{tabular}

$B M I$ body mass index

deviation (SD) if it was normally distributed or was expressed as median followed with interquartile range if the data was skewed. The RIs were estimated by the IBM SPSS Statistic 21 (SPSS Inc., Chicago, IL, USA, RRID:SCR_002865) using the nonparametric approach. To examine the statistical significance of GlyA levels between any two groups, the Student's t test was used and $P<0.05$ was considered to be statistically significant.
The correlation between GlyA and FPG, 1 h-, 2 h- postprandial plasma glucose was determined separately by the Pearson's correlation coefficient test. The chi-square test was performed to compare the incidence rates of greater-than-upper-limit for GlyA levels in the third trimester between the groups with and without adverse pregnancy outcomes. The odds ratio (OR) was applied to evaluate the association between the adverse

\section{A}

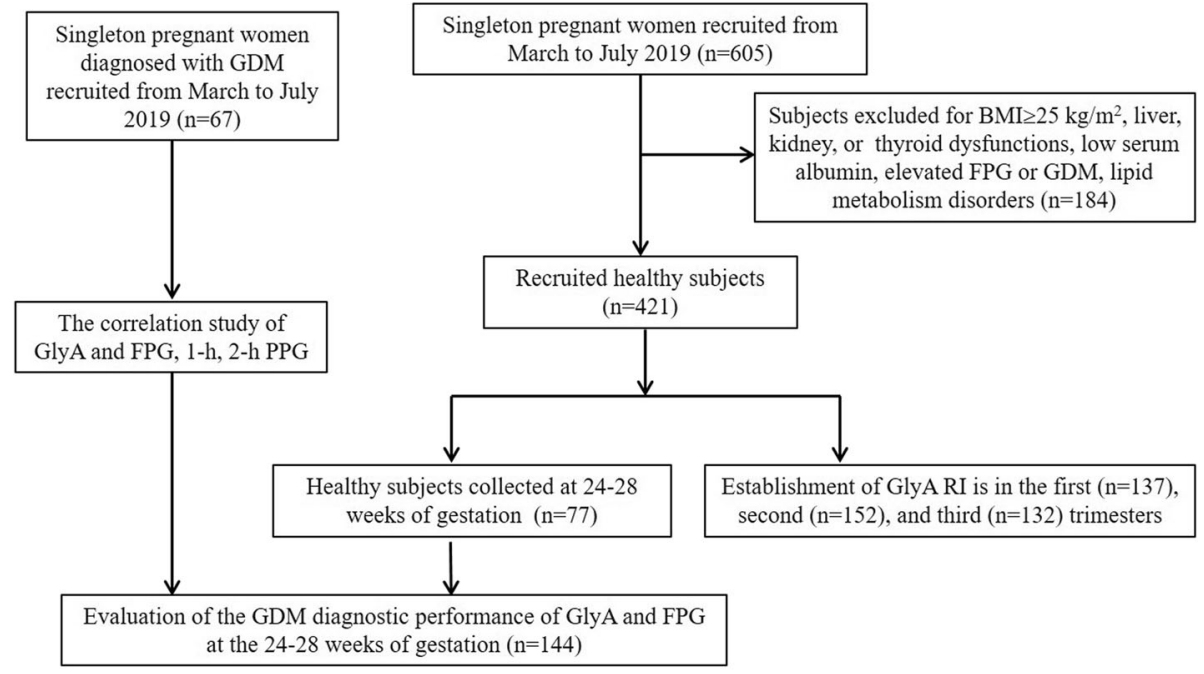

\section{B}

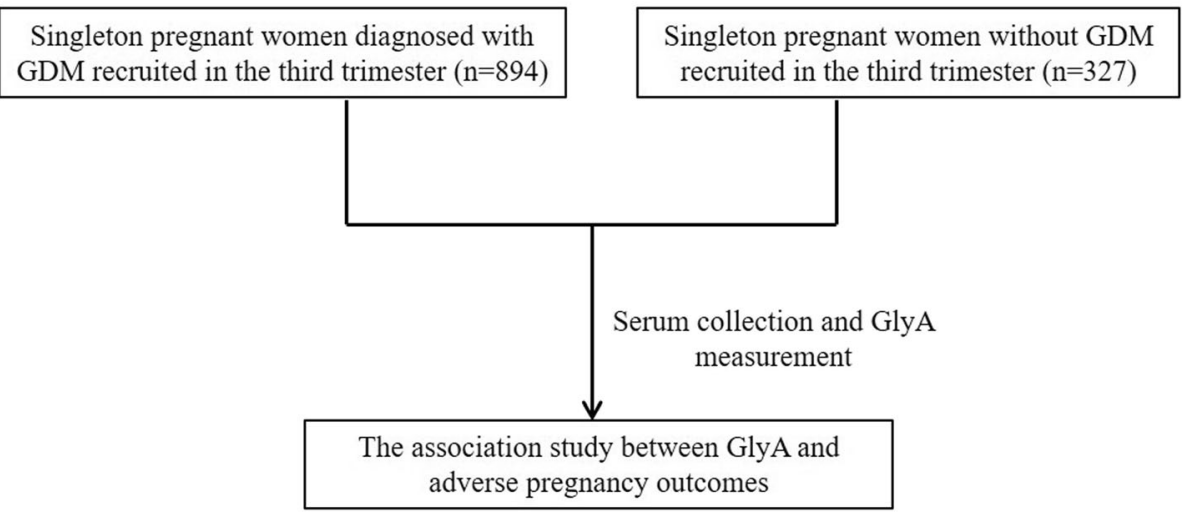

Fig. 1 Schematic diagram for patient recruitment and study design. a the study design for GlyA reference intervals (RIs) establishment and its evaluation in GDM diagnosis; $\mathbf{b}$ the study design for the associations between third trimester GlyA and adverse pregnancy outcomes. FPG, fasting plasma glucose; PPG, postprandial plasma glucose 
outcomes and the elevation of GlyA. The receiver operating characteristic (ROC) curve analysis was performed to compare the diagnostic power between GlyA and fasting plasma glucose at the 24-28 weeks of gestation for GDM and assess the predictive value of GlyA in adverse pregnant outcomes in women with and without GDM.

\section{Results}

The ages of subjects, the pre-pregnancy BMI and the weeks of gestation were summarized in Table 1 . There was no significant difference for maternal ages and pre-pregnancy BMI when compared between different trimesters.

With normal distributions examined by the KolmogorovSmirnov test (data not shown), the means of the GlyA level were $13.22 \pm 0.98 \%$ in the first trimester (less than 13 weeks), $11.89 \pm 0.88 \%$ in the second trimester (14-27 weeks), and $11.33 \pm 0.86 \%$ in the third trimester ( $28-40$ weeks). According to the CLSI guideline EP28-A3C (original GlyA data available in the Additional file 1: Table S1), the 2.5th and 97.5th were used as the lower and upper limits of RIs respectively. The $90 \%$ confidence interval (CI) of the lower and upper limits was calculated with bootstrap method. As shown in Table 2, the RIs of GlyA were 11.26-15.10\%, $10.06-13.51 \%$, and $9.76-13.09 \%$ in the first, second and third trimesters respectively.

As shown in Fig. 2, the GlyA level was significant lower in the second trimester than that in the first trimester $(P<0.001)$. This decreasing tendency was also recognized when comparing the GlyA in the second with that in the third trimesters $(P<0.001)$.

Interestingly, there was no statistical difference of the GlyA levels between the GDM group and the healthy pregnancy at 24-28 weeks of gestation (Fig. 3) (Additional file 1: Table S2). When compared with the GlyA, the FPG had a better discriminating power for GDM, with the areas under curve (AUC) of $0.705(P<0.001)$ in the ROC analysis (Fig. 4). Furthermore, the GlyA level did not show a linear correlation with the FPG $\left(\mathrm{R}^{2}=\right.$ $0.021 ; P=0.245), 1$-h $\left(R^{2}=0.002 ; P=0.711\right)$ or 2 -h postprandial plasma glucose $\left(R^{2}=0.013 ; P=0.349\right)$ in women with GDM at 24-28 weeks of gestation.

In the association study with the GDM patients, the third trimester GlyA level did not exhibit a higher incidence rate of greater-than-upper-limit (Gly A > 13.09\%) in the patients with preterm delivery, hypertension, $\mathrm{PE}$ or LGA, when compared with the control group

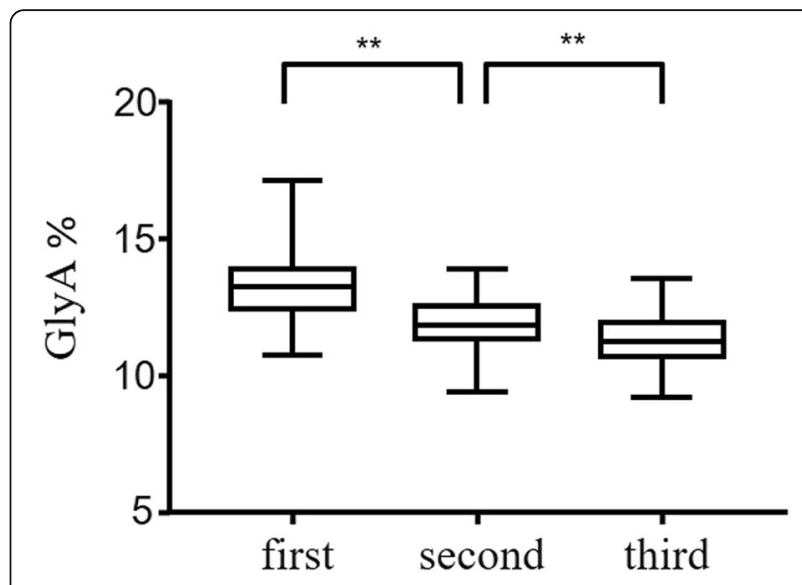

Fig. 2 Box plots of glycated albumin in the healthy pregnant women recruited in present study. *indicates $P<0.05$; ${ }^{* *}$ indicates $P<0.001$. Student's t test was performed to calculate the $P$ value

(Table 3). The exception was a made in the patients with postpartum hemorrhage, in which higher GlyA level was observed $(P=0.03, \mathrm{OR}=1.57$, Table 3$)$. In addition, the GlyA showed better discriminating power by ROC curves $($ AUC $=0.663)$ (Fig. 5) in the patients with postpartum hemorrhage than in the patients with the rest adverse outcomes (ROC data not shown). By contrast, in the analyses with the non-GDM patients, the GlyA was not statistically different in the patients with or without adverse pregnancy outcomes.

\section{Discussion}

The GlyA is considered a more sensitive blood glucose indicator than the HbAlc in pregnancy with a shorter half-life of around 2-3 weeks [7, 17]. Kouzuma et al. [18] reported that the GlyA reference interval in the non-pregnant Americans with normal glucose tolerance was $11.9-15.8 \%$. However, with the observation made in present study, the GlyA level was gradually decreased when the gestation age went up. This physiological change of serum GlyA level during pregnancy has been reported both in healthy pregnant women and in those diagnosed with GDM [11]. Hiramatsu et al. [8] showed that the normal ranges of GlyA in healthy Japanese women were $12.2-16.6 \%$ in first trimester, $11.8-15.6 \%$ in second trimester and $11.3-15.5 \%$ in third trimester with both lower and upper limits higher than those reported in our study, suggesting the lack of the GlyA

Table 2 Trimester-specific reference intervals of glycated albumin

\begin{tabular}{llll}
\hline & First trimester & Second trimester & Third trimester \\
\hline GlyA(\%), mean \pm SD & $13.22 \pm 0.98$ & $11.89 \pm 0.88$ & $11.33 \pm 0.86$ \\
$2.5 \%$ percentile $(90 \% \mathrm{Cl})$ & $11.26(10.88,11.77)$ & $10.06(9.57,10.43)$ & $9.76(9.37,9.99)$ \\
$97.5 \%$ percentile $(90 \% \mathrm{Cl})$ & $15.10(14.68,16.30)$ & $13.51(13.26,13.85)$ & $13.09(12.69,13.43)$
\end{tabular}

GlyA glycated albumin, SD standard deviation, $\mathrm{Cl}$ confidence interval 


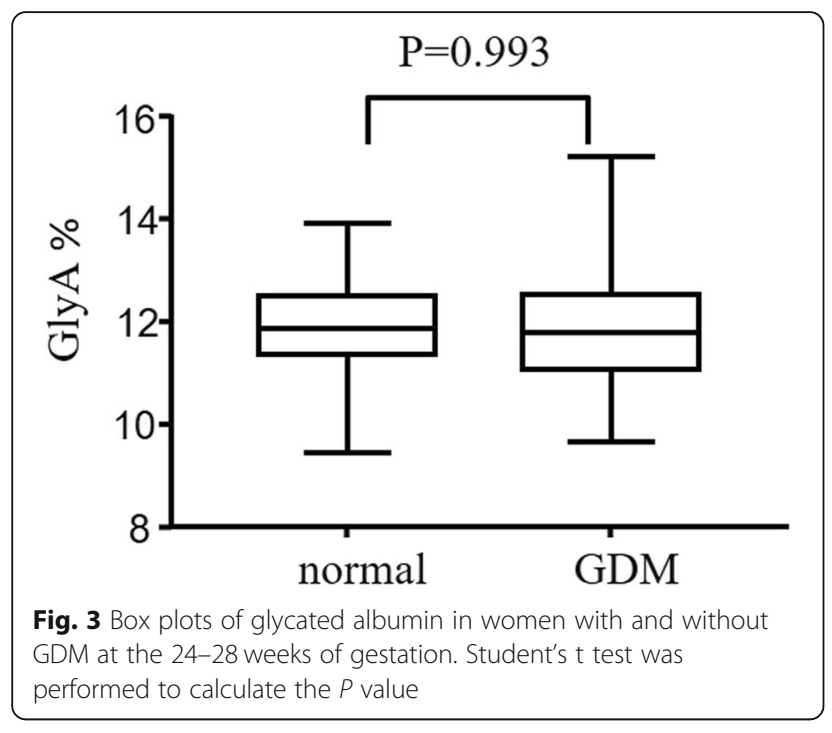

reagent uniformity and the potential impact of different ethnic background. Even with the same GlyA reagent (Lucica GlyA-L, Asahi Kasei, Tokyo) that was used by the above Japanese research group, the mean GlyA value of the 24-28 weeks gestations with Chinese pregnant women was still lower than that of the Japanese pregnant women [11].
Both BMI and urinary protein has been reported as two important factors influencing GlyA levels during pregnancy. The GlyA concentrations were found much lower in the high BMI group $\left(\geq 25 \mathrm{~kg} / \mathrm{m}^{2}\right)$ than in the low BMI group $\left(18.5-25 \mathrm{~kg} / \mathrm{m}^{2}\right)$; the GlyA was also lower in the pregnant women with elevated urinary protein [8]. Selvin et al. [19] also reported an inverse association between GlyA and BMI. However, the underlying mechanism for decreased GlyA level in the subjects with higher BMI still remains unknown. One hypothesis was that the chronic inflammation related turnover of negative acute-phase proteins might have led to the decrease of GlyA [20]. Glomerular filtration rate (GFR) could also be attributed to the changes of GlyA level during pregnancy. It has been reported that the GlyA level was inversely increased when the estimated GFR (eGFR) was decreased due to renal dysfunction in both diabetic and nondiabetic population [21]. As pregnancy progressed, physiologically elevated eGFR may also result in the decrease of GlyA.

We compared the GlyA levels of pregnant women with and without GDM at the 24-28 weeks of gestation and found no significant difference $(P=0.993)$ between the two groups. This result was consistent with a study conducted by Zhu et al. [22], in which no statistical GlyA difference was observed between the patients with

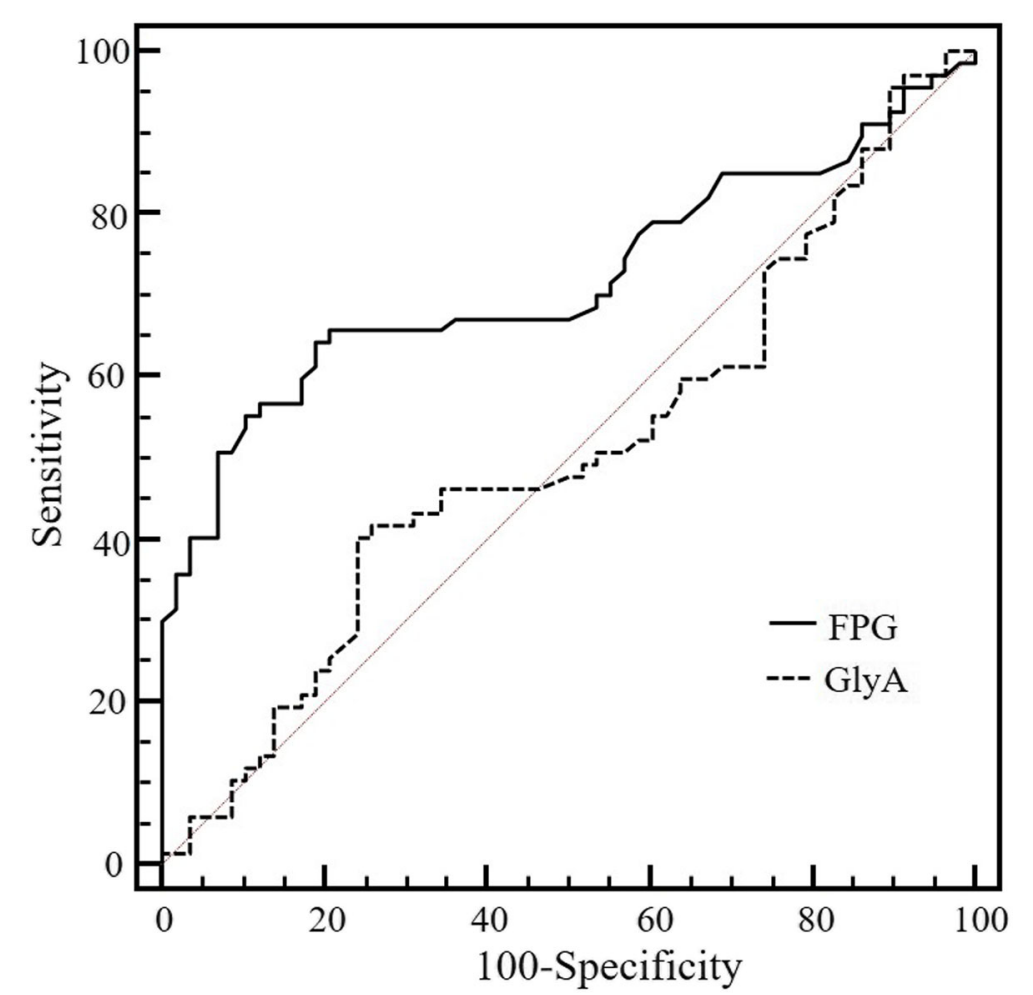

Fig. 4 Receiver operating characteristic (ROC) curve analysis of GlyA and FPG at the 24-28 weeks of gestation for diagnosing GDM. The areas under ROC curve were 0.503, $(P=0.957)$ for GlyA and $0.705(P<0.001)$ for FPG 
Table 3 Comparisons of glycated albumin of pregnant women with and without adverse pregnancy outcomes

\begin{tabular}{lllll}
\hline & $\begin{array}{l}\text { Case } \\
\text { N/total }\end{array}$ & $\begin{array}{l}\text { Control } \\
\text { N/total }\end{array}$ & Odds ratio & P-value \\
\hline Non-GDM group $(n=327)$ & & & & \\
Preterm delivery & $0 / 5$ & $4 / 322$ & 0.00 & 1.00 \\
Postpartum hemorrhage & $0 / 14$ & $4 / 313$ & 0.00 & 1.00 \\
Preeclampsia & $0 / 16$ & $4 / 311$ & 0.00 & 1.00 \\
Hypertension & $0 / 69$ & $4 / 258$ & 0.00 & 0.58 \\
LGA & $0 / 12$ & $4 / 310$ & 0.00 & 1.00 \\
GDM group ( $n=894)$ & & & & \\
Preterm delivery & $13 / 63$ & $212 / 831$ & 0.76 & 0.40 \\
Postpartum hemorrhage & $37 / 160$ & $118 / 734$ & 1.57 & 0.03 \\
Preeclampsia & $10 / 60$ & $215 / 834$ & 0.58 & 0.12 \\
Hypertension & $9 / 45$ & $180 / 849$ & 0.93 & 0.85 \\
LGA & $31 / 114$ & $181 / 717$ & 1.11 & 0.66 \\
\hline
\end{tabular}

case: patients with the specific adverse pregnancy outcome; control: patients without specific adverse pregnancy outcome. LGA: large for gestation age. N: the number of subjects with GlyA greater-than-upper-limit (13.09\%). total: the total number of the subjects with (case) or without (control) specific adverse pregnancy outcome. Chi-square test was performed for $P$ value calculation. For the LGA study, the subjects with preterm delivery were excluded

and without GDM. In current practice, GDM was diagnosed during 24-28 weeks of gestations by FPG and postprandial plasma glucose levels. As the HbA1c concentration was influenced by the half-life of red blood cell which could be prolonged by iron-deficiency during pregnancy, its application in GDM diagnosis has not been widely recommended [23, 24]. As a non-traditional glycemic marker, the limited diagnostic value of GlyA in GDM has been reported in several articles. Zhu et al. [22] reported that the AUC values were 0.726 for FPG and only 0.542 for GlyA in the second trimester. Similar observation was made by Saglam et al. [25], with the AUC of GlyA being 0.550 in the GDM diagnosis. In our study, we also found a higher diagnostic value of FPG (AUC $=0.721)$ than that of GlyA (AUC $=0.509)$ during the 24-28 weeks of gestation. Besides, there was no linear correlation between the GlyA and the FPG, 1-h or 2$\mathrm{h}$ postprandial plasma glucose (PPG) with the collected serum samples. However, Koga et al. [26] reported that GlyA could accurately reflect the postprandial plasma glucose. Huang et al. [27] showed that the FPG and the GlyA values exhibited a significant correlation in all pregnant women although the linear coefficient was only 0.103 . In a non-pregnant population including subjects with diabetes mellitus, impaired glucose regulation and normal glucose regulation, the linear correlation between GlyA and FPG had been observed with a better coefficient factor $\left(R^{2}=0.41\right)$ and GlyA was also observed statistically correlated with 2-h PPG $(\mathrm{R} 2=0.43)$ [28]. Interestingly, a study with the euglycaemia group showed that the GlyA levels did not correlate with PPG and was likely to reflect incidental glycation throughout the albumin lifespan [29]. Plus, as a distinct clinical

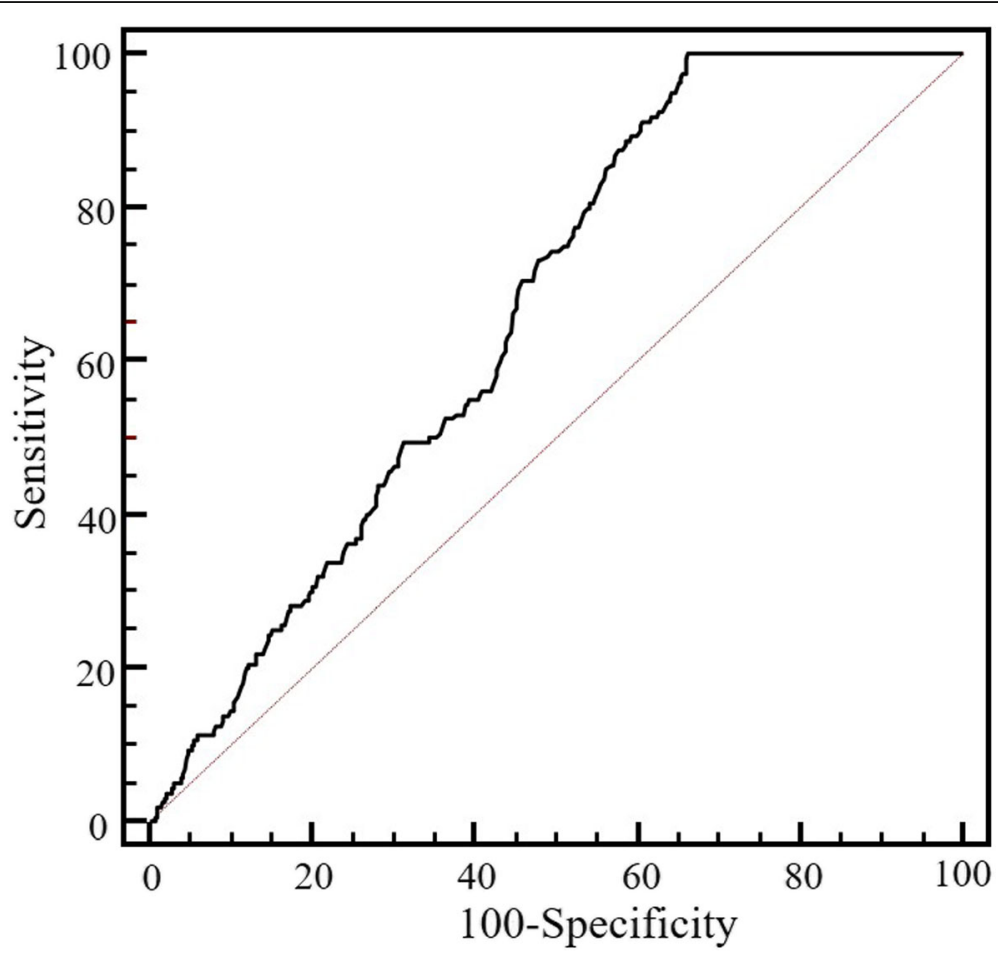

Fig. 5 Receiver operating characteristic (ROC) curve analysis of GlyA for predicting postpartum hemorrhage in women with GDM 
entity, GDM is at least partially introduced by the profound physiological changes during pregnancy, such as hormones or mediators secreted by placenta. And in some sense, GDM could be seen as the early stage of type-2 diabetes mellitus [30]. Therefore, together with the insufficient GDM discrimination power in our study, we hypothesize that the GlyA formation in GDM patients may behave more like that in euglycemic women and could not well predict FPG or PPG response.

Fetal macrosomia which is defined as infant birth weight above a specific threshold, historically $4000 \mathrm{~g}$ or $4500 \mathrm{~g}$, is a common adverse neonatal outcome of GDM. With the threshold of $4000 \mathrm{~g}$, the incident rate of macrosomia is $15-45 \%$ of women diagnosed with GDM compared with $12 \%$ of normal women [31]. The Pedersen's hypothesis explaining the pathophysiology of macrosomia is that the maternal hyperglycemia leads to fetal hyperglycemia and hyperinsulinemia which further result in protein and fat stores in fetus [31]. However, due to lack of standard diagnosis criteria for macrosomia, LGA has been alternatively used to assess excessive fetal growth based on the population birthweight curves. Here we explored the association between GlyA and birth weight to evaluate the third trimester GlyA in predicting LGA. Our results showed that the GlyA was not significantly different (with GlyA= $13.09 \%$ as threshold, Table 3) in the patients with or without LGA in both GDM and non-GDM groups (Table 2). Interestingly, Zhang et al. [32] conducted a study involving 242 Chinese pregnant women with GDM and they found the GlyA level had no association with neonate birth weight in the late pregnancy. Another study with Chinese women diagnosed with GDM showed that the GlyA level at 36-38 weeks of gestation had no difference between the maternal group with birth weight of 3000-3499 g and the group with birth weight of 3500-3999 g [11]. In a multicenter study including 136 Japanese diabetic pregnant women, the incidence of large-for-date showed no statistical difference between the group of GlyA $\geq 15.8 \%$ group and the group of GlyA $<15.8 \%$ group with $P=0.071$ [13]. However, the above negative findings about the GlyA prediction on birth weight have been controversial. In a study of 42 Japanese women with GDM, the maternal GlyA level was significantly higher in the group of infants with large-for-date status [33]. It has been also reported that an average increase of $76.1 \mathrm{~g}$ in birth weight was observed per $1 \%$ maternal GlyA elevation [10]. According to the work by Catalano et al. [34], although no significant difference of the infant birth weight between the GDM and the non-GDM groups was found, the fat mass of infants was changed in the same direction as the maternal blood glucose level. Therefore, the blood glucose or GlyA may have a better predictive value for neonatal body compositions (such as fat mass) than simple body weight.
Unfortunately, to our best knowledge, the previous publication that was directly focused the association between GlyA level in late pregnancy and maternal adverse outcomes was limited. In a recent study by Kumari et al. with Indian population, the prevalence of postpartum hemorrhage in GDM and non-GDM groups was not significantly different [35]. Interestingly, a Chinese group reported that poor glycemic control monitored by FPG in the GDM patients had led to elevated postpartum hemorrhage [36]. Similarly, our data also showed that in the GDM group with postpartum hemorrhage more patients had elevated GlyA, suggesting the importance of glycemic control in late pregnancy. It has been relatively well established that GDM posed an increased risk of hypertension in patients of different ethnic backgrounds including Chinese population [37-39]. In contrast, whether the rate of preterm delivery increases in pregnancies complicated with GDM remained debatable [40]. Nevertheless, elevated GlyA did not seem to aggravate the prevalence of hypertension or preterm delivery in the patients with or without GDM (Table 3).

Although it was not statistically different, lower incidence rate of great-than-upper-limit for the GlyA level was observed with the GDM patients who suffered from preeclampsia $(10 / 60=16.7 \%$ in the PE case group vs 215 / $834=25.8 \%$ in the control group, $\mathrm{OR}=0.58$ ) (Table 3). In the study focused on the relationship between the serum albumin and the preeclampsia, it was found that women in the preeclampsia group displayed significantly lower level of serum albumin than those in the normal group. It was proposed that albumin might function in suppressing vascular oxidative stress and preventing endothelial dysfunction [41]. Therefore, the decreased albumin and the resulting decreased GlyA may have made the patients more vulnerable to preeclampsia. In another relevant research, Wang et al. reported that glycated serum protein (measured as serum fructosamine) was decreased in $\mathrm{PE}$ patient during pregnancy [42].

\section{Conclusions}

The trimester-specific RIs of GlyA showed an obvious decreasing trend throughout the entire pregnancy. As a short-term glycemic control indicator, the GlyA level has limited value in diagnosing GDM at the 24-28 weeks of gestation and in predicting adverse pregnancy outcomes.

\section{Supplementary information}

Supplementary information accompanies this paper at https://doi.org/10. 1186/s12884-019-2704-x.

Additional file 1. The original glycated albumin and glucose measurements in the trimester reference intervals establishment and the GDM diagnosis study. Table S1. GlyA data of healthy pregnant women in three trimesters. Table S2. GlyA and FPG data of women with and without GDM at the 24-28 weeks of gestation. 


\section{Abbreviations}

AUC: Areas under ROC curve; BMI: Body mass index; Cl: Confidence interval; CLSI: Clinical and Laboratory Standards Institute; FPG: Fasting plasma glucose; GDM: Gestational diabetes mellitus; GFR: Glomerular filtration rate; GlyA: Glycated albumin; HbA1c: Glycated hemoglobin A1c; LGA: Large for gestational age; OGTT: Oral glucose tolerance test; PE: Preeclampsia; Rl: Reference interval; ROC: Receiver operating characteristic

\section{Acknowledgements}

Not applicable.

\section{Authors' contributions}

All authors have certified the author list and the contribution description. All authors have read and approved the submitted manuscript and any substantially modified version of the manuscript. Contribution to work: $Y D$ $Y Z, J W, Y C, X X, C Z, J L, Y L, G T, L H$ and $Z C$ were involved in conception and design of the study, performing experiments, acquisition of data, analysis and interpretation of data; YD and ZC were involved in drafting of the article and critical approval of the final article; $Y D, L L$, and $Z C$ were involved in the statistical analysis and figure preparation.

\section{Funding}

This study was supported by National Natural Science Foundation of China (No.81702057). The funding body did not take part in the design of the study, the collection, analysis and interpretation of the data, or writing the manuscript.

\section{Availability of data and materials}

The original GlyA and FPG datasets generated during the current study are available and provided as Additional file 1: Table S1 and Table S2.

Alternatively, the Additional file are available in the Open Science Framework Repository (www.osf.io, DOl https://doi.org/10.17605/OSF.IO/AN6R4). However, according the patients' verbal consents, their biometrics and pregnancy outcomes are only available from the corresponding author on reasonable request.

\section{Ethics approval and consent to participate}

This study was approved by the Ethics Committee of Beijing Obstetrics and Gynecology Hospital, Capital Medical University (approval number: 2017-KY070-01). The verbal consents from the participants were required as no clinical intervention was involved, which was approved by the ethical committee of our institute.

\section{Consent for publication}

Not applicable.

\section{Competing interests}

The authors declare that they have no competing interests.

\section{Author details}

'Department of Laboratory Medicine, Beijing Obstetrics and Gynecology Hospital, Capital Medical University, 251 Yaojiayuan Road, Beijing 100026, China. ${ }^{2}$ Obstetrical Department, Beijing Obstetrics and Gynecology Hospital, Capital Medical University, Beijing, China. ${ }^{3}$ Prenatal Diagnosis Center, Beijing Haidian Maternal and Child Health Hospital, Beijing, China. ${ }^{4}$ Central Laboratory, Beijing Obstetrics and Gynecology Hospital, Capital Medical University, Beijing, China.

Received: 21 November 2019 Accepted: 24 December 2019 Published online: 03 January 2020

\section{References}

1. American Diabetes A. Standards of medical Care in Diabetes-2017: summary of revisions. Diabetes Care. 2017;40:S4-5 https://doi.org/10.2337/dc17-S003.

2. Caissutti C, Berghella V. Scientific evidence for different options for GDM screening and management: controversies and review of the literature. Biomed Res Int. 2017;2017:2746471 https://doi.org/10.1155/2017/2746471.

3. Lavery JA, Friedman AM, Keyes KM, Wright JD, Ananth CV. Gestational diabetes in the United States: temporal changes in prevalence rates between 1979 and 2010. BJOG. 2017;124:804-13 https://doi.org/10.1111/ 1471-0528.14236.
4. Rodrigo N, Glastras SJ. The emerging role of biomarkers in the diagnosis of gestational diabetes mellitus. J Clin Med. 2018;7 https://doi.org/10.3390/ jcm7060120.

5. O'Keeffe DT, Maraka S, Rizza RA. HbA1c in the evaluation of diabetes mellitus. JAMA. 2016;315:605-6 https://doi.org/10.1001/jama.2015.16561.

6. Colagiuri S, Lee CM, Wong TY, Balkau B, Shaw JE, Borch-Johnsen K, et al. Glycemic thresholds for diabetes-specific retinopathy: implications for diagnostic criteria for diabetes. Diabetes Care. 2011;34:145-50 https://doi. org/10.2337/dc10-1206.

7. Freitas PAC, Ehlert LR, Camargo JL. Glycated albumin: a potential biomarker in diabetes. Arch Endocrinol Metab. 2017;61:296-304 https://doi.org/10. 1590/2359-3997000000272.

8. Yuji H, Ikki S, Yasue O, Masao N. Determination of reference intervals of glycated albumin and hemoglobin A1c in healthy pregnant Japanese women and analysis of their time courses and influencing factors during pregnancy. Endocr J. 2012;59:145-51.

9. Phelps RL, Honig GR, Green D, Metzger BE, Frederiksen MC, Freinkel N. Biphasic changes in hemoglobin A1C concentrations during normal human pregnancy. Am J Obstet Gynecol. 1983;147:651-3 https://doi.org/10.1016/ 0002-9378(83)90443-X.

10. Mendes N, Alves M, Andrade R, Ribeiro RT, Papoila AL, Serrano F. Association between glycated haemoglobin, glycated albumin and fructosamine with neonatal birthweight and large-for-date status infants in gestational diabetes mellitus: a prospective cohort study. J Obstet Gynaecol. 2019;39:768-73 https://doi.org/10.1080/01443615.2019.1584886.

11. Li HP, Wang FH, Tao MF, Huang YJ, Jia WP. Association between glycemic control and birthweight with glycated albumin in Chinese women with gestational diabetes mellitus. J Diabetes Investig. 2016;7:48-55 https://doi. org/10.1111/jdi.12383.

12. Sugawara D, Sato H, Ichihashi K, Nagai K, Kawano A. Glycated albumin level during late pregnancy as a predictive factor for neonatal outcomes of women with diabetes. J Matern Fetal Neonatal Med. 2018;31:2007-12 https://doi.org/10.1080/14767058.2017.1333103.

13. Shimizu I, Hiramatsu Y, Omori Y, Nakabayashi M, Group JGAS. Comparison of HbA1c and glycated albumin as a control marker for newborn complications in diabetic women in a multicentre study in Japan (Japan glycated albumin study group: study 2). Ann Clin Biochem. 2018;55:639-46 https://doi.org/10.1177/0004563218763695.

14. Goda A, Masuyama T. Obesity and Overweight in Asian People. Circ J. 2016; 80:2425-6 https://doi.org/10.1253/circj.CJ-16-1087.

15. Clinical and Laboratory Standard Institute. Defining, Establishing, and Verifying Reference Intervals in the Clinical Laboratory; Approved Guideline, 3rd edn. 2008

16. International Association of $D$, Pregnancy Study Groups Consensus $P$, Metzger BE, Gabbe SG, Persson B, Buchanan TA, et al. International association of diabetes and pregnancy study groups recommendations on the diagnosis and classification of hyperglycemia in pregnancy. Diabetes Care. 2010;33:676-82 https://doi.org/10.2337/dc09-1848.

17. Zhou Q, Shi DB, Lv LY. The establishment of biological reference intervals of nontraditional glycemic markers in a Chinese population. J Clin Lab Anal. 2017;31 https://doi.org/10.1002/jcla.22097.

18. Kohzuma T, Yamamoto T, Uematsu Y, Shihabi ZK, Freedman BI. Basic performance of an enzymatic method for glycated albumin and reference range determination. J Diabetes Sci Technol. 2011;5:1455-62 https://doi.org/ $10.1177 / 193229681100500619$

19. Selvin E, Warren B, He X, Sacks DB, Saenger AK. Establishment of community-based reference intervals for Fructosamine, Glycated albumin, and 1,5-Anhydroglucitol. Clin Chem. 2018;64:843-50 https://doi.org/10.1373/ clinchem.2017.285742

20. Koga M, Otsuki M, Matsumoto S, Saito H, Mukai M, Kasayama S. Negative association of obesity and its related chronic inflammation with serum glycated albumin but not glycated hemoglobin levels. Clin Chim Acta. 2007; 378:48-52 https://doi.org/10.1016/j.cca.2006.10.013.

21. Duan N, Zhu SN, Li HX, Jiao LL, Yang HY, Guo Q. Assessment of Glycated albumin as a useful Indicator for renal dysfunction in diabetic and nondiabetic population. Clin Lab. 2017;63:1129.

22. Zhu J, Chen Y, Li C, Tao M, Teng Y. The diagnostic value of glycated albumin in gestational diabetes mellitus. J Endocrinol Investig. 2018;41:1218 https://doi.org/10.1007/s40618-016-0605-7.

23. American Diabetes A. Standards of medical care in diabetes--2014. Diabetes Care. 2014;37(Suppl 1):S14-80 https://doi.org/10.2337/dc14-S014. 
24. Diagnostic criteria and classification of hyperglycaemia first detected in pregnancy: a World Health Organization Guideline. Diabetes Res Clin Pract. 2014;103:341-63. https://doi.org/10.1016/j.diabres.2013.10.012.

25. Saglam B, Uysal S, Sozdinler S, Dogan OE, Onvural B. Diagnostic value of glycemic markers HbAIC, 1,5-anhydroglucitol and glycated albumin in evaluating gestational diabetes mellitus. Ther Adv Endocrinol Metab. 2017;8: 161-7 https://doi.org/10.1177/2042018817742580.

26. Koga M. Glycated albumin; clinical usefulness. Clin Chim Acta. 2014;433:96104 https://doi.org/10.1016/j.cca.2014.03.001.

27. Huang $Y, H u$ Y, Ma YU, Ye G. Glycated albumin is an optimal biomarker for gestational diabetes mellitus. Exp Ther Med. 2015;10:2145-9 https://doi.org/ 10.3892/etm.2015.2808

28. Ma XJ, Pan JM, Bao YQ, Zhou J, Tang JL, Li Q, et al. Combined assessment of glycated albumin and fasting plasma glucose improves the detection of diabetes in Chinese subjects. Clin Exp Pharmacol Physiol. 2010;37:974-9 https://doi.org/10.1111/j.1440-1681.2010.05417.x.

29. Reynolds AN, Duncan A, Kruimer D, Venn BJ. Glycated albumin is associated with body mass index in euglycemic adults but is not predictive of postprandial blood glucose response. J Clin Lab Anal. 2017;31:1-4 https:// doi.org/10.1002/jcla.22085.

30. Chiefari E, Arcidiacono B, Foti D, Brunetti A. Gestational diabetes mellitus: an updated overview. J Endocrinol Investig. 2017;40:899-909 https://doi.org/10. 1007/s40618-016-0607-5.

31. Kc K, Shakya S, Zhang H. Gestational diabetes mellitus and macrosomia: a literature review. Ann Nutr Metab. 2015;66(Suppl 2):14-20 https://doi.org/10. $1159 / 000371628$

32. Zhang YC, Ni YH, Ji X, Ying CM. Correlation of neonate birth weight with glycated hemoglobin A1c and glycated albumin levels in patients with gestational diabetes mellitus. Lab Med. 2017;32:165-8 [in Chinese].

33. Sugawara D, Maruyama A, Imanishi T, Sugiyama Y, Ichihashi K.

Complications in infants of diabetic mothers related to Glycated albumin and hemoglobin levels during pregnancy. Pediatr Neonatol. 2016;57:496500 https://doi.org/10.1016/j.pedneo.2016.02.003.

34. Catalano PM, Thomas A, Huston-Presley L, Amini SB. Increased fetal adiposity: a very sensitive marker of abnormal in utero development. Am J Obstet Gynecol. 2003;189:1698-704 https://doi.org/10.1016/s00029378(03)00828-7.

35. Kumari R, Dalal V, Kachhawa G, Sahoo I, Khadgawat R, Mahey R, et al. Maternal and perinatal outcome in gestational diabetes mellitus in a tertiary Care Hospital in Delhi. Indian J Endocrinol Metab. 2018;22:116-20 https:// doi.org/10.4103/ijem.JJEM_582_17.

36. Gao H. The association between glycemic control and pregnancy outcomes in 90 gestational diabetes mellitus. Matern Child Health Care China. 2012;27: 1650-2 [in Chinese].

37. Tobias DK, Hu FB, Forman JP, Chavarro J, Zhang C. Increased risk of hypertension after gestational diabetes mellitus: findings from a large prospective cohort study. Diabetes Care. 2011;34:1582-4 https://doi.org/10. 2337/dc11-0268

38. Wang L, Leng J, Liu H, Zhang S, Wang J, Li W, Li W, Li N, Zhang T, Baccarelli AA, et al. Association between hypertensive disorders of pregnancy and the risk of postpartum hypertension: a cohort study in women with gestational diabetes. J Hum Hypertens. 2017;31:725-30 https://doi.org/10.1038/jhh.2017.46.

39. Vanlalhruaii, Ranabir S, Prasad L, Singh NN, Singh TP. Prevalence of gestational diabetes mellitus and its correlation with blood pressure in Manipuri women. Indian J Endocrinol Metab. 2013;17:957-61 https://doi org/10.4103/2230-8210.122597

40. Yogev $Y$, Langer $\mathrm{O}$. Spontaneous preterm delivery and gestational diabetes: the impact of glycemic control. Arch Gynecol Obstet. 2007;276:361-5 https://doi.org/10.1007/s00404-007-0359-8.

41. Kinoshita H, Watanabe K, Azma T, Feng GG, Akahori T, Hayashi H, et al. Human serum albumin and oxidative stress in preeclamptic women and the mechanism of albumin for stress reduction. Heliyon. 2017;3:e00369 https://doi.org/10.1016/j.heliyon.2017.e00369.

42. Wang $X$, Zhang $R$, Zhang $L$, Tian Z. Glycated serum proteins: high in pancreatic cancer and low in preeclampsia. Prog Mol Biol Transl Sci. 2019; 162:321-33 https://doi.org/10.1016/bs.pmbts.2019.01.007.

\section{Publisher's Note}

Springer Nature remains neutral with regard to jurisdictional claims in published maps and institutional affiliations.

\section{Ready to submit your research? Choose BMC and benefit from:}

- fast, convenient online submission

- thorough peer review by experienced researchers in your field

- rapid publication on acceptance

- support for research data, including large and complex data types

- gold Open Access which fosters wider collaboration and increased citations

- maximum visibility for your research: over $100 \mathrm{M}$ website views per year

At $\mathrm{BMC}$, research is always in progress.

Learn more biomedcentral.com/submissions 